OPEN ACCESS

Edited by:

Kevin R. Brooks,

Macquarie University, Australia

Reviewed by:

Adar Pelah,

University of York, United Kingdom

Donald Alfred Owens,

Franklin \& Marshall College,

United States

${ }^{*}$ Correspondence:

Martina Carament martina.caramenti@ibfm.cnr.it

${ }^{\dagger}$ These authors have contributed equally to this work

Specialty section:

This article was submitted to

Perception Science,

a section of the journal

Frontiers in Psychology

Received: 04 June 2019 Accepted: 01 October 2019 Published: 16 October 2019

Citation:

Caramenti M, Pretto $P$,

Lafortuna CL, Bresciani J-P and Dubois A (2019) Influence of the Size of the Field of View on Visual Perception While Running in a

Treadmill-Mediated Virtual Environment.

Front. Psychol. 10:2344. doi: 10.3389/fpsyg.2019.02344

\section{Influence of the Size of the Field of View on Visual Perception While Running in a Treadmill-Mediated Virtual Environment}

\author{
Martina Caramenti ${ }^{1,2,3 *}$, Paolo Pretto ${ }^{4}$, Claudio L. Lafortuna ${ }^{5}$, Jean-Pierre Bresciani ${ }^{1,6+}$ \\ and Amandine Dubois $1,7+$ \\ ${ }^{1}$ Department of Neurosciences and Movement Sciences, University of Fribourg, Fribourg, Switzerland, ${ }^{2}$ Istituto di \\ Bioimmagini e Fisiologia Molecolare, Consiglio Nazionale delle Ricerche, Segrate, Italy, ${ }^{3}$ HumanTech Institute, University of \\ Applied Sciences and Arts Western Switzerland, Fribourg, Switzerland, ${ }^{4}$ Virtual Vehicle Research Center, Graz, Austria, \\ ${ }^{5}$ Istituto di Fisiologia Clinica, Consiglio Nazionale delle Ricerche, Milan, Italy, ${ }^{6}$ LPNC, University Grenoble Alpes, Grenoble, \\ France, ${ }^{7}$ Université de Lorraine, 2LPN-CEMA Group (Cognition-EMotion-Action), EA 7489, Metz, France
}

We investigated how the size of the horizontal field of view (FoV) affects visual speed perception with individuals running on a treadmill. Twelve moderately trained to trained participants ran on a treadmill at two different speeds (8 and $12 \mathrm{~km} / \mathrm{h}$ ) in front of a moving virtual scene. Different masks were used to manipulate the visible visual field, masking either the central or the peripheral area of the virtual scene or showing the full visual field. We asked participants to match the visual speed of the scene to their actual running speed. For each trial, participants indicated whether the scene was moving faster or slower than they were running. Visual speed was adjusted according to the responses using a staircase method until the Point of Subjective Equality was reached, that is until visual and running speed were perceived as matching. For both speeds and all FoV conditions, participants underestimated visual speed relative to the actual running speed. However, this underestimation was significant only when the peripheral FoV was masked. These results confirm that the size of the FoV should absolutely be taken into account for the design of treadmill-mediated virtual environments (VEs).

Keywords: virtual reality, visual speed perception, treadmill running, field of view, optical flow, locomotion

\section{INTRODUCTION}

Because of the associated physiological and psychological benefits, physical activity (PA) is fundamental for human health and well-being. Physical activity notably helps to reduce the risk of many diseases and improving the functional status and the quality of life. Treadmills constitute one of the most widely used pieces of equipment to train cardiovascular fitness indoors. However, treadmills have some shortcomings. Specifically, treadmill locomotion is monotonous and can easily lead to boredom. Moreover, it fails to reproduce overground locomotion because it is characterized by a sensory discrepancy between kinesthetic/motor and visual information (Pelah and Barlow, 1996). Coupling treadmills and virtual environments (VEs) could contribute to improve the engagement and effort of the user, and to enhance the physical experience. In addition, it would help minimizing the kinesthetic-visual discrepancy. 
Though coupling fitness equipment with VEs likely represents the future of cardiovascular training equipment, some issues must still be resolved. This is notably the case regarding motion perception. In particular, when moving around an environment, perceived body motion results from the integration of motion cues provided by different sensory channels. Visual and proprioceptive cues, together with efference copy information, are among the most prominent sensory inputs in this integration process. Regarding more specifically treadmill-mediated VEs, though one might legitimately assume that visual speed should match locomotion speed, i.e., the speed at which the belt is moving, studies have repeatedly shown that walkers/runners tend to underestimate optical flow speed relative to locomotion speed (Thurrell et al., 1998; Banton et al., 2005; Durgin et al., 2005a; Thurrell and Pelah, 2005; Kassler et al., 2010; Powell et al., 2011; Caramenti et al., 2018). In particular, these studies have shown that visual speed needs to be set higher than the actual walking/running speed to be perceived as matching.

For devices that should reach a wide number of users, such as treadmills, an additional related problem regards the size of the display, and more particularly the horizontal field of view (FoV). Specifically, several studies have shown that the size of the FoV affects visual speed perception, the underestimation of which tends to increase as the FoV decreases (Van Veen et al., 1998; Nilsson et al., 2014, 2017). These studies were performed for different motion-related activities, such as walking, cycling, or simulated driving. For instance, Van Veen et al. (1998) found the visual perception of cycling speed to depend on the peripheral FoV. In this study, visual speed was underestimated when the FoV was smaller than 73 degrees, and slightly overestimated when the FoV was larger than 107 degrees. With individuals walking at $3 \mathrm{mph}(4,83 \mathrm{~km} / \mathrm{h})$ and gazing straight ahead, Banton et al. (2005) found a 50\% underestimation of walking speed with a FoV of 50 degrees. In the same study, walking speed was accurately perceived when peripheral flow was maximized with downward or sideways gaze (Banton et al., 2005). With a 90-degree FoV, Thurrell et al. (1998), Thurrell and Pelah (2002) found that a $3 \mathrm{mph}(4,83 \mathrm{~km} / \mathrm{h})$ walking speed is perceived about $20 \%$ slower than it actually is, i.e., it is underestimated by $20 \%$. Note that when the FoV reduces, visual speed underestimation tends to increase. In particular, with a FoV of about 90 degrees, visual gains (i.e., visual speed/locomotor speed) that are perceived as natural range from 1.67 to 2.03, whereas with a FoV of 25 degrees, the visual gains that are perceived as natural rather range from 2.14 to 2.64 (Nilsson et al., 2014). Taken together, these results suggest that while walking, visual speed is perceived more accurately as peripheral flow increases. Similar results were observed with static individuals. In particular, in an experiment in which perceived visual speed was investigated with seating individuals, Pretto et al. (2009) found that at FoVs smaller than 60 degrees, visual speed is underestimated with a bias that is inversely related to the size of the visible area. Interestingly, these authors also found that visual speed tends to be overestimated if the optical flow is presented in peripheral vision only. Note that in this study, the horizontal FoV was 220 degrees and optical flow was also provided on the floor (quarter sphere screen).
Surprisingly, to date, no study has investigated how the size of the FoV affects visual speed perception with running participants. Yet, in order to design treadmill-mediated visual environments for a widespread diffusion, it is fundamental to understand how the display dimensions could affect perceived speed, notably so that the user can experience the best possible perceptual coherence.

Here we used a virtual environment and psychophysics methods to assess how the size of the FoV affects the perceived speed of the optical flow with participants running on a treadmill.

\section{MATERIALS AND METHODS}

Twelve moderately trained to trained subjects ( 1 female, 11 male) with a median age of $25.53(26.04 \pm 2.37$ SD) participated in the study. All were naïve about the purpose of the study, had normal or corrected-to-normal vision and none had a history of cardiovascular disease. All participants gave their informed and written consent prior to the inclusion in the study, which was performed in accordance with the ethical standards specified by the 1964 Declaration of Helsinki. This study was approved by the Ethics Committee of the University of Fribourg.

Before performing the running task, the participants filled out the short version of the International Physical Activity Questionnaire (IPAQ - French version), which estimates the weekly amount of PA expressed in MET-min/week. METs, or metabolic equivalents, are defined as the ratio between the energy rate expended during a specific activity and the energy expenditure at rest (1MET). In other words, METs describe the energy expenditure of an activity. The volume of PA per week is calculated as the sum of the METs expended in different activities over a week, taking into account the time in minutes spent in each activity. This volume is expressed as MET-min/week.

Participants ran on a treadmill (HP Cosmos Mercury Running surface: $150 \mathrm{~cm}$ by $50 \mathrm{~cm}$ ) located in front of a $430 \mathrm{~cm} \times 270 \mathrm{~cm}$ projection screen. A $2 \mathrm{D}$ virtual scene representing an open-air hallway was projected onto the screen (Projector: Barco F50 WUXGA, Resolution: $1920 \times 1200$ pixel) to simulate optical flow (see Figure 1A). The virtual scene was created using Unity 3D (Unity Technologies). When running, the participants' head was located $300 \mathrm{~cm}$ from the center of the screen, with an effective FoV of $70^{\circ}$ in full screen mode. The room was darkened during the experiment, with the display screen being the only source of light. While running, participants held a custom-made plastic cylinder $(115 \mathrm{~mm} \times 30 \mathrm{~mm}, 15 \mathrm{~g})$ in each hand, with a response button located on the top surface. This allowed participants to send their responses via Bluetooth to the computer by effortlessly pressing on the buttons with their thumb while running.

The experiment consisted of six blocks. In each block, the participants ran at one of two different speeds (i.e., treadmill speed), namely $8 \mathrm{~km} / \mathrm{h}$ or $12 \mathrm{~km} / \mathrm{h}$. For each treadmill speed, three different FoV conditions were presented. Specifically, different masks were implemented in the visual scene to manipulate the visible area shown on the screen. Note that a fixation cross positioned at the end of the hallway was visible 
A

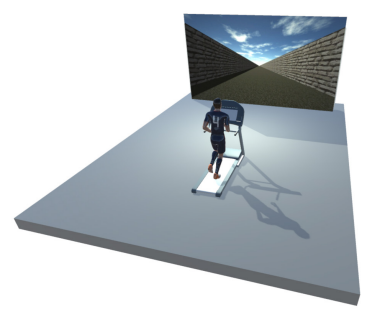

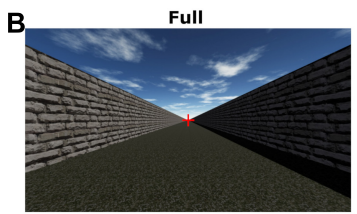

Central

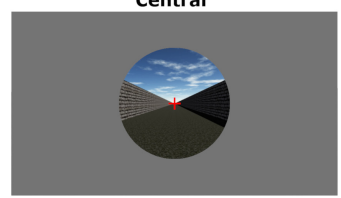

Peripheral

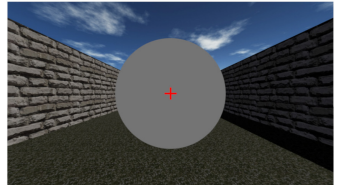

C

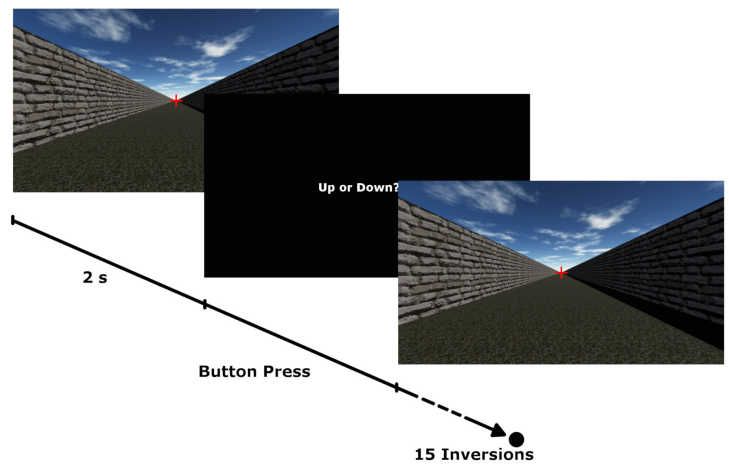

FIGURE 1 | Experimental setup (A), experimental conditions (B) and experimental design (C).

for the whole duration of the trials. The fixation cross was located at the focus of expansion of the optical flow resulting from the relative motion between the visual scene and the observer (i.e., participants). In the full FoV condition, the openair hallway was visible on the whole screen. In the central FoV condition, the hallway was displayed only within a circular mask of $30^{\circ}$ centered on the fixation cross. Finally, in the peripheral FoV condition, a disk covered the central portion of the screen $\left(30^{\circ}\right)$ so that optical flow was visible only in the outer area of the screen, i.e., in peripheral vision (see Figure 1B). The order of presentation of the six blocks was counterbalanced between subjects using a Latin square.

For each FoV condition, the participants were presented with the visual scene while they were running at constant speed on the treadmill. For each trial, the task was to estimate if the visual scene was slower or faster than the actual running speed. Specifically, participants were instructed to gaze at the fixation cross that was visible at the end of the virtual hallway. The visual scene was presented for $2 \mathrm{~s}$ before the participants were asked the question "Up or down?" displayed on a black screen:

- "Up" if they perceived visual speed to be slower than the treadmill, requiring an increase of the speed of the virtual scene - left response button.

- "Down" if they perceived visual speed to be faster than the treadmill, requiring a decrease of the speed of the virtual scene - right response button.

Participants gave their response by pressing the corresponding switch while continuing to run on the treadmill. Once the participant pressed the response button, the graphics returned from the black screen to the visual scene of the following trial (see Figure 1C). Luminance values were $6.7 \mathrm{~cd} / \mathrm{m}^{2}$ for the visual scene used as the main stimulus and $0.2 \mathrm{~cd} / \mathrm{m}^{2}$ for the black screen. For each trial, the speed of the visual scene was adjusted with a one up one down staircase method (Leek, 2001; Kingdom and Prins, 2010) that took into account the previous responses of the participant. The staircase defined an increase/decrease of the visual speed of $0.5 \mathrm{Km} / \mathrm{h}$ until the first inversion of the response, followed by steps of $0.3 \mathrm{Km} / \mathrm{h}$. When 15 inversions of the responses were reached the staircase session was stopped. This method was used to determine the Point of Subjective Equality (PSE), namely a perceptual threshold indicating the respective values of two quantities when they are perceived as being equivalent. For each running speed, the PSE indicated the speed of the visual scene (optical flow) that was perceived as matching the actual running speed. For each of the two running speeds, two consecutive staircase sessions were proposed in a random order, the staircase session starting once with a higher $(+4 \mathrm{~km} / \mathrm{h})$ and once with a lower $(-4 \mathrm{~km} / \mathrm{h})$ visual speed than the treadmill speed. At the end of each staircase session, the participants could take a short pause before starting the next staircase session.

Prior to the experiment, the participants had a few minutes to familiarize themselves with treadmill running at different running speeds. Using a training program, they were then familiarized with the experimental setting and task. The training program presented the visual scene at different speeds. Experimental trials were initiated when the participant felt comfortable running at different speeds.

\section{RESULTS}

For each FoV condition (i.e., Full screen, Peripheral view, Central view) and each treadmill speed (i.e., 8 and $12 \mathrm{~km} / \mathrm{h}$ ), perceived visual speed was compared to the actual running speed using either a one-sample $t$-test or a Wilcoxon signed-rank test (when perceived visual speed data was not normally distributed). Please refer to Supplementary Table $\mathbf{S 1}$ for the data. After assessing normality using a Shapiro-Wilk test, the one-sample $t$-test was used when perceived visual speed data was normally distributed, 


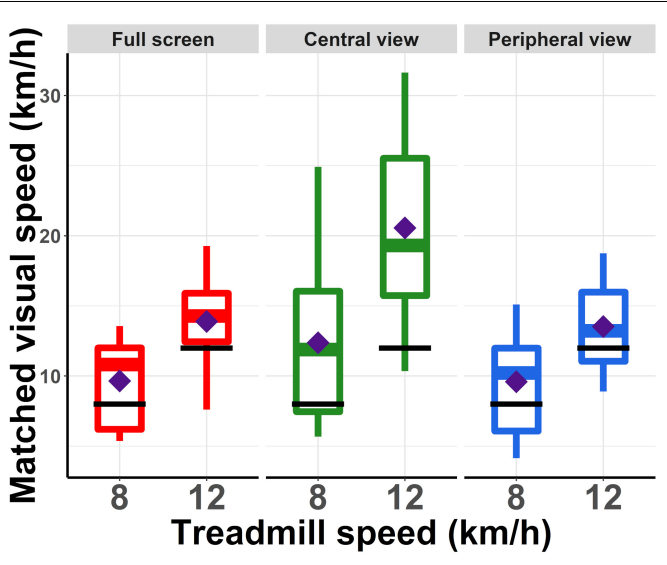

FIGURE 2 | Speed of the visual scene that was perceived as matching treadmill speed. In all conditions for most participants and on average (filled diamonds), the visual scene had to move faster than the actual treadmill speed (black lines) to be perceived as matched. while the Wilcoxon signed-rank test was used for not-normally distributed data. For all six tests, the alpha level was corrected for multiple comparisons using Holm correction. For all six conditions, the visual speed was set higher than the actual running speed, meaning that the optical flow had to move faster than the treadmill speed for the two speeds to be perceived as matching, indicating that visual speed was underestimated relative to running speed. However, this underestimation was significant only in the Central view condition at a running speed of $12 \mathrm{~km} / \mathrm{h}(t(11)=4.323, p=0.001)$. It also barely failed to reach significance in the Central view condition at a running speed of $8 \mathrm{~km} / \mathrm{h}(t(11)=2.666, p=0.02$ uncorrected, with an alpha adjusted to 0.01 ). For the other four conditions, there was no significant difference between the set visual speed and the actual treadmill speed. Figure 2 shows the average matching visual speed for the three FoV conditions and the two treadmill speeds.

We then tested whether there were significant differences across conditions regarding the amplitude of relative underestimation of visual speed (see Figure 3). For each FoV condition and each treadmill speed, we computed the percentage of visual under/overestimation using the equation: In (perceived visual speed/actual treadmill speed)*100. This percentage indicates how much slower (negative values) or faster (positive values) the optical flow had to move relative to the actual running speed, i.e., treadmill speed, for the two speeds to be perceived as equivalent. The logarithm (i.e., $\ln$ ) was used so that neither visual nor treadmill speed was used as an absolute reference value (Graff, 2014).

The mean relative underestimation values measured in the different conditions were compared using a linear mixed model. We used a linear mixed model because we had a repeated measures design and data was non-parametric. The analysis revealed a main effect of the FoV $\left(\chi^{2}(2)=33.50, p<0.0001\right)$. In particular, visual speed underestimation was larger for the Central view condition (41.30\%) than for the Full screen (12.32\%) and Peripheral view condition (10.22\%). There was

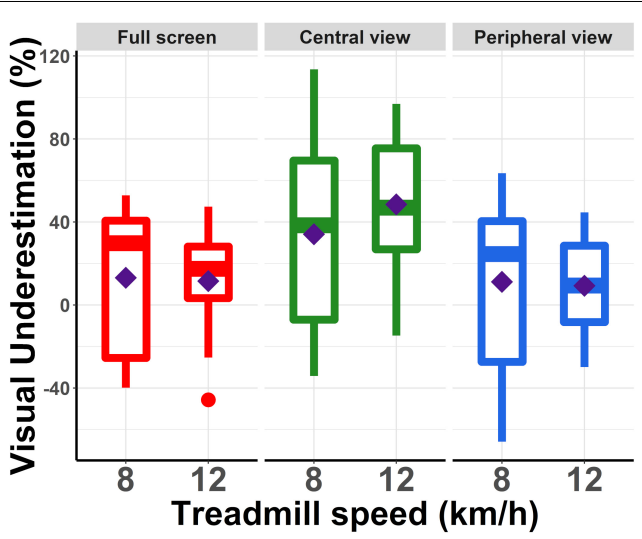

FIGURE 3 | Percentage of underestimation of visual speed relative to running speed. These values were computed using the equation: In (perceived visual speed/actual running speed) * 100. Each box summarized for each running speed and each condition the distribution of the responses of the participants. The diamond corresponds to the mean value. The central line corresponds to the median, with the box defining the inter-quartile range (IQR) between the first and the third quartile and the whiskers corresponding to $\pm 1.5 \mathrm{IQR}$.

no main effect of the treadmill speed $\left(\chi^{2}(1)=1.02, p=0.31\right)$, and visual underestimation varied from $19.45 \%$ at $8 \mathrm{~km} / \mathrm{h}$ to $23.11 \%$ at $12 \mathrm{~km} / \mathrm{h}$.

Planned orthogonal contrasts indicated that the visual underestimation observed in the Central view condition was of significantly larger amplitude than those observed in the Full screen $(p=0.003)$ and Peripheral view condition $(p=0.0014)$, respectively. On the other hand, the amplitude of visual underestimation was not significantly different between the Full screen and the Peripheral view condition $(p=0.76)$.

Because the linear mixed model analysis revealed no significant effect of treadmill speed, we collapsed the two treadmill speed levels and compared the three FoV conditions using a Friedman Rank Sum test (repeated measures and nonparametric data). The analysis indicated a main effect of the FoV on visual speed underestimation $\left(\chi^{2}(2)=15.167, p<0.001\right)$, thereby confirming the outcome of the linear mixed model analysis. Post hoc tests confirmed a significant difference between the Central view and the other two conditions, whereas the Full screen and Peripheral view conditions did not differ from one another (see Figure 4).

\section{DISCUSSION}

While running on a treadmill in front of a moving virtual scene, participants were asked to match the visual speed of the scene to their running speed (i.e., treadmill speed) in different FoV conditions. Previous studies have shown that the size of the FoV affects speed perception, but this had never been tested with running subjects. The results of the current study show that for all three FoV conditions, namely Full screen, Peripheral view and Central view, and both running speeds tested in the experiment (i.e., 8 and $12 \mathrm{~km} / \mathrm{h}$ ), visual speed tended to be underestimated 


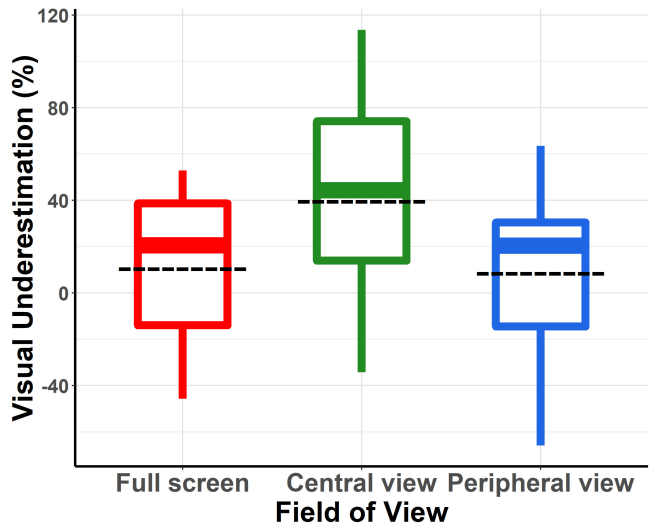

FIGURE 4 | Percentage of underestimation of visual speed relative to running speed in the three FoV conditions (when collapsing the two running speeds). These values were computed using the equation: In (perceived visual speed/actual running speed) * 100. Each box summarized for each running speed and each condition the distribution of the responses of the participants. The dashed line corresponds to the mean value. The central line corresponds to the median, with the box defining the inter-quartile range (IQR) between the first and the third quartile and the whiskers corresponding to $\pm 1.5 \mathrm{IQR}$.

with respect to running speed. Specifically, the speed of the virtual scene was systematically set higher than the actual running speed in order to be perceived as matching treadmill speed. This relative underestimation of visual speed was significant only in the Central view condition at a running speed of $12 \mathrm{~km} / \mathrm{h}$, while it barely failed to reach significance after correction for multiple comparisons in the Central view condition at a running speed of $8 \mathrm{~km} / \mathrm{h}$.

The visual underestimation (relative to running speed) we observed in the Central View condition is significantly larger than the underestimation observed in the Full screen and in the Peripheral view condition. This is in line with the results reported in previous studies performed with cycling (Van Veen et al., 1998) and walking individuals (Thurrell et al., 1998; Thurrell and Pelah, 2002; Banton et al., 2005), but also with sitting-still participants (Pretto et al., 2009). When moving forward, the angular velocity of the optical flow is relatively low in the part of the visual field directly surrounding the focus of expansion. In most situations, and definitely in our experiment, this area is the central part of the visual field. In contrast, the angular velocity of the optical flow is much higher in peripheral vision because this usually corresponds to an area that is farther to the focus of expansion. In line with this, the underestimation of visual speed that we observed in the Central view condition likely results from the fact that in this condition, only the low angular velocity component of the optical flow was visible. This confirms that the extent of the FoV should absolutely be considered when designing VEs.

Several studies have suggested that peripheral vision is important for motion perception, affecting speed perception (Pretto et al., 2009, 2012), navigation abilities (Czerwinski et al., 2002; Turano et al., 2005), and the sensation of self-motion deriving from a moving stimulus (Brandt et al., 1973; Berthoz et al., 1975; Mohler et al., 2005). Peripheral vision has also been shown to play an important role in evoking vection (i.e., the subjective experience of self-motion without actual self-motion) (Brandt et al., 1973; Berthoz et al., 1975). In their 2009 study, Pretto et al. (2009) found that when the central part of the visual field was masked (in the range 10 to 60 degrees, which is the range also tested in our experiment), the speed of the presented optical flow was systematically overestimated. In contrast to their observations, we did not find any overestimation of visual speed in the Peripheral view condition, and this condition did not differ from the Full screen condition. This difference between their results and ours could be linked to the dimensions of the screen (i.e., FoV) used in the two experiments. In particular, Pretto et al. (2009) used a quarter sphere screen with a $230^{\circ}$ horizontal $\times 125^{\circ}$ vertical FoV, with projection on the floor. This is much larger than the projection screens used in most studies as well as than the maximum FoV of head mounted displays. The results of Van Veen (Van Veen et al., 1998) seem to confirm this hypothesis, since this author also found a slight overestimation of the speed of the optical flow when the FoV was larger than $107^{\circ}$.

An additional difference between our study and that of Pretto et al. (2009) is the motion-related sensory information that was available during the simulated body motion. In our experiment, participants were running on a treadmill. Therefore, in addition to the optical flow, participants were provided with motionrelated proprioceptive and efference copy information. The task of the participants was actually to compare visual information with proprioceptive and efference copy information. As opposed to this, in the study of Pretto et al. (2009), participants were sitting still. In other words, in their study, optical flow was the only source of motion information. This difference between the two studies might explain, at least partially, why Pretto and colleagues observed an overestimation of visual speed in the Peripheral FoV condition, whereas we did not. Specifically, in our experiment, proprioceptive and efferent copy information might have prevented participants from overestimating visual speed by indicating them that they were not running that fast. Several studies have shown that visual, proprioceptive and vestibular information is used to update the representation of the visual space for an efficient navigation, providing congruent information about the displacement (Warren and Hannon, 1988; Loomis et al., 1996, 1999; Chance et al., 1998; Yardley and Higgins, 1998; Sun et al., 2004a; Turano et al., 2005; Jürgens and Becker, 2006; Souman et al., 2009). In fact, studies have shown that when either one of visual, vestibular or proprioceptive information is missing during locomotion, it can be predicted based on the other two sensory channels (Mittelstaedt and Mittelstaedt, 2001; Durgin and Gigone, 2007; Durgin, 2009). For the perception of visual speed, evidence suggests that optical flow speeds near to walking speeds are better discriminated when one is walking (Durgin et al., 2004; Durgin, 2009), but it is not clear if vision or proprioception is more important for monitoring self-motion speed when both are available (Sun et al., 2004b). This means that the manipulation of visual, vestibular or proprioceptive information can influence the perception of self-motion and the related motor activity (Durgin et al., 2005b; Mohler et al., 2007; Pelah et al., 2015).

A last difference between our study and that of Pretto et al. (2009) relates to the experimental design. Specifically, Pretto and colleagues used a Two-interval forced choice (2 IFC) in which the 
Full screen condition constituted the reference and was used as standard stimulus. In other words, perceived visual speed in the other FoV conditions was always measured relative to perceived visual speed in the Full screen condition. In contrast to that, in our experiment, perceived visual speed was compared to the actual running speed. In theory, this methodological difference should not have a strong impact on the difference between the Full screen and the other FoV conditions. However, and in line with the above-mentioned arguments, speed comparison was visuo-visual in Pretto et al. (2009), whereas it was visuoproprioceptive + efference copy in our experiment. This might have affected the way optical flow information was processed.

Previous studies with walking (Banton et al., 2005; Durgin et al., 2005a; Kassler et al., 2010; Powell et al., 2011) and running participants (Caramenti et al., 2018, 2019) reported a general tendency to underestimate visual speed when compared to locomotor speed. Our results confirm the tendency to underestimate visual speed, as the mean visual-locomotor gain observed in our study ranged from $1.31: 1$ at $8 \mathrm{~km} / \mathrm{h}$ to $1.34: 1$ at $12 \mathrm{~km} / \mathrm{h}$. One factor that could have affected the visual-locomotor gain is treadmill running in itself, which has been shown to influence speed perception. In fact, treadmill locomotion is usually perceived as faster than the corresponding overground speed (White et al., 1998; Marsh et al., 2006; Kong et al., 2009, 2012), with differences in running kinematics and kinetics that have been shown to affect the capacity to discriminate the actual running speed (Nelson et al., 1972; Nigg et al., 1995; Wank et al., 1998; Riley et al., 2008). Contrary to previous studies performed with running individuals (Caramenti et al., 2018, 2019), here we did not find a speed-dependent modulation of the visual-locomotor gain. This could be due to the high PA levels of the participants, which were estimated with the IPAQ questionnaire (MET-min/week: $3956 \pm 1777$ ). In fact, PA seems to modulate visual speed perception with individuals running in treadmill-mediated VEs (Caramenti et al., 2019). In particular, previous studies have shown that when used to move within a range of velocities, people might associate sensory signals related to self-motion (biomechanical and vestibular information, optical flow) to their actual locomotion speed more adequately (Bredin et al., 2005). This could mean that regular exposure to visual flow at certain speeds might help to better calibrate the mapping between motion-evoked visual information and the corresponding kinesthetic and motor information.

Another factor that might have contributed to the underestimation of visual speed is the lack of stereoscopic information. Specifically, some studies have shown that stereopsis can provide information about relative distance not only in the peripersonal space but also at greater observation distances (Palmisano et al., 2010), and that perceived depth tends to be more "compressed" under monocular viewing conditions (Allison et al., 2009). Moreover, stereoscopic cues seem to enhance the precision of perceived speed, thereby helping to estimate the rate of self-motion (Brooks and Rafat, 2015) and the perceived speed of vection (Palmisano et al., 2019).

\section{CONCLUSION}

Treadmill-mediated VEs may soon become a useful and broadly used tool to enhance people's engagement and adherence to PA programs. Our results indicate that the design of such environments should definitely take into account the size of the FoV in order to reduce the discrepancy between visual and kinesthetic/efferent information during treadmill locomotion, so as to provide the most natural and engaging feedback possible.

\section{DATA AVAILABILITY STATEMENT}

All datasets generated for this study are included in the manuscript/Supplementary Files.

\section{ETHICS STATEMENT}

The studies involving human participants were reviewed and approved by Internal Review Board of the Department of Psychology of the University of Fribourg. The patients/participants provided their written informed consent to participate in this study.

\section{AUTHOR CONTRIBUTIONS}

MC, PP, J-PB, AD, and CL designed the experiments. MC and $\mathrm{AD}$ performed the experiments. $\mathrm{MC}, \mathrm{AD}$, and $\mathrm{JB}$ performed the statistical analysis. MC wrote the first draft of the manuscript. All authors contributed to the manuscript revision, read, and approved the submitted version.

\section{ACKNOWLEDGMENTS}

The authors thank Rudy Mazzocchi (Department of Neuroscience and Movement Science, University of Fribourg, Fribourg, Switzerland) for recruiting the participants and for providing support in data collection, Marco Scodeggio (Istituto di Bioimmagini e Fisiologia Molecolare, Consiglio Nazionale delle Ricerche, Segrate, Milano, Italy) for technical assistance in constructing the response device, and Thibaut Le Naour (Department of Neuroscience and Movement Science, University of Fribourg, Fribourg, Switzerland) for his help in the creation of the representation of the experimental set-up (Figure 1A).

\section{SUPPLEMENTARY MATERIAL}

The Supplementary Material for this article can be found online at: https://www.frontiersin.org/articles/10.3389/fpsyg. 2019.02344/full\#supplementary-material 


\section{REFERENCES}

Allison, R. S., Gillam, B. J., and Vecellio, E. (2009). Binocular depth discrimination and estimation beyond interaction space. J. Vis. 9, 10-10. doi: 10.1167/9.1.10

Banton, T., Stefanucci, J., Durgin, F., Fass, A., and Proffitt, D. R. (2005). The perception of walking speed in a virtual environment. Presence 14, 394-406. doi: 10.1162/105474605774785262

Berthoz, A., Pavard, B., and Young, L. R. (1975). Perception of linear horizontal self-motion induced by peripheral vision (linearvection) basic characteristics and visual-vestibular interactions. Exp. Brain Res. 23, 471-489.

Brandt, T., Dichgans, J., and Koenig, E. (1973). Differential effects of central versus peripheral vision on egocentric and exocentric motion perception. Exp. Brain Res. 16, 476-491. doi: 10.1007/bf00234474

Bredin, J., Kerlirzin, Y., and Israël, I. (2005). Path integration: is there a difference between athletes and non-athletes? Exp. Brain Res. 167, 670-674. doi: 10.1007/ s00221-005-0251-3

Brooks, K. R., and Rafat, M. E. (2015). Simulation of driving in low-visibility conditions: does stereopsis improve speed perception? Perception 44, 145-156. doi: $10.1068 / \mathrm{p} 7779$

Caramenti, M., Lafortuna, C. L., Mugellini, E., Khaled, O. A., Bresciani, J.-P., and Dubois, A. (2018). Matching optical flow to motor speed in virtual reality while running on a treadmill. PLoS One 13:e0195781. doi: 10.1371/journal.pone. 0195781

Caramenti, M., Lafortuna, C. L., Mugellini, E., Khaled, O. A., Bresciani, J.-P., and Dubois, A. (2019). Regular physical activity modulates perceived visual speed when running in treadmill-mediated virtual environments. PLoS One 14:e0219017. doi: 10.1371/journal.pone.0219017

Chance, S. S., Gaunet, F., Beall, A. C., and Loomis, J. M. (1998). Locomotion mode affects the updating of objects encountered during travel: the contribution of vestibular and proprioceptive inputs to path integration. Presence 7, 168-178. doi: 10.1162/105474698565659

Czerwinski, M., Tan, D. S., and Robertson, G. G. (2002). "Women take a wider view," in Proceedings of the SIGCHI Conference on Human Factors in Computing Systems, New York, NY.

Durgin, F. H. (2009). When walking makes perception better. Curr. Dir. Psychol. Sci. 18, 43-47. doi: 10.1111/j.1467-8721.2009.01603.x

Durgin, F. H., Fox, L. F., Schaffer, E., and Whitaker, R. (2005a). "The perception of linear self-motion," in Proceedings of the Human Vision and Electronic Imaging $X$ (2005), San Jose, CA.

Durgin, F. H., Pelah, A., Fox, L. F., Lewis, J., Kane, R., and Walley, K. A. (2005b). Self-motion perception during locomotor recalibration: more than meets the eye. J. Exp. Psychol. Hum. Percept. Perform. 31, 398-398. doi: 10.1037/00961523.31.3.398

Durgin, F. H., and Gigone, K. (2007). Enhanced optic flow speed discrimination while walking: contextual tuning of visual coding. Perception 36, 1465-1475. doi: $10.1068 / \mathrm{p} 5845$

Durgin, F. H., Gigone, K. M., and Schaffer, E. (2004). Improved visual speed discrimination while walking. J. Vis. 4, 802-802. doi: 10.1167/4.8.802

Graff, C. (2014). Expressing relative differences (in percent) by the difference of natural logarithms. J. Math. Psychol. 60, 82-85. doi: 10.1016/j.jmp.2014.02.001

Jürgens, R., and Becker, W. (2006). Perception of angular displacement without landmarks: evidence for bayesian fusion of vestibular, optokinetic, podokinesthetic, and cognitive information. Exp. Brain Res. 174, 528-543. doi: 10.1007/s00221-006-0486-487

Kassler, L., Feasel, J., Lewek, M. D., Brooks, F. P. Jr., and Whitton, M. C. (2010). "Matching actual treadmill walking speed and visually perceived walking speed in a projection virtual environmen," in Proceedings of the 7th Symposium on Applied Perception in Graphics and Visualization, Los Angeles, CA.

Kingdom, F. A. A., and Prins, N. (2010). Psychophysics: A Practical Introduction. Amsterdam: Elsevier Science.

Kong, P. W., Candelaria, N. G., and Tomaka, J. (2009). Perception of self-selected running speed is influenced by the treadmill but not footwear. Sports Biomech. 8, 52-59. doi: 10.1080/14763140802629990

Kong, P. W., Koh, T. M. C., Tan, W. C. R., and Wang, Y. S. (2012). Unmatched perception of speed when running overground and on a treadmill. Gait Posture 36, 46-48. doi: 10.1016/j.gaitpost.2012.01.001

Leek, M. R. (2001). Adaptive procedures in psychophysical research. Percept. Psychophys. 63, 1279-1292. doi: 10.3758/bf03194543
Loomis, J. M., Da Silva, J. A., Philbeck, J. W., and Fukusima, S. S. (1996). Visual perception of location and distance. Curr. Dir. Psychol. Sci. 5, 72-77. doi: 10. 1111/1467-8721.ep10772783

Loomis, J. M., Klatzky, R. L., Golledge, R. G., and Philbeck, J. W. (1999). "Human navigation by path integration," in Wayfinding Behavior: Cognitive Mapping and Other Spatial Processes, ed. R. G. Golledge (Baltimore, MD: Johns Hopkins University Press), 125-151.

Marsh, A. P., Katula, J. A., Pacchia, C. F., Johnson, L. C., Koury, K. L., and Rejeski, W. J. (2006). Effect of treadmill and overground walking on function and attitudes in older adults. Med. Sci. Sports Exerc. 38, 1157-1157.

Mittelstaedt, M.-L., and Mittelstaedt, H. (2001). Idiothetic navigation in humans: estimation of path length. Exp. Brain Res. 139, 318-332. doi: 10.1007/ s002210100735

Mohler, B. J., Thompson, W. B., Creem-Regehr, S. H., Pick, H. L. Jr., and Warren, W. H. Jr. (2007). Visual flow influences gait transition speed and preferred walking speed. Exp. Brain Res. 181, 221-228. doi: 10.1007/s00221-0070917-0

Mohler, B. J., Thompson, W. B., Riecke, B., and Bülthoff, H. H. (2005). "Measuring vection in a large screen virtual environment," in Proceedings of the 2nd Symposium on Applied Perception in Graphics and Visualization, A Coruña.

Nelson, R. C., Dillman, C. J., Lagasse, P., and Bickett, P. (1972). Biomechanics of overground versus treadmill running. Med. Sci. Sports 4:233. doi: 10.1249/ 00005768-197200440-00029

Nigg, B. M., De Boer, R. W., and Fisher, V. (1995). A kinematic comparison of overground and treadmill running. Med. Sci. Sports Exerc. 27, 98-105.

Nilsson, N. C., Nordahl, R., and Serafin, S. (2017). "Waiting for the ultimate display: can decreased fidelity positively influence perceived realism," in Proceedings of the 2017 IEEE 3rd Workshop on Everyday Virtual Reality (WEVR), Los Angeles, CA.

Nilsson, N. C., Serafin, S., and Nordahl, R. (2014). Establishing the range of perceptually natural visual walking speeds for virtual walking-in-place locomotion. IEEE Trans. Vis. Comput. Graph. 20, 569-578. doi: 10.1109/TVCG. 2014.21

Palmisano, S., Davies, R. G., and Brooks, K. R. (2019). Vection strength increases with simulated eye-separation. Atten. Percept. Psychophys. 81, 281-295. doi: 10.3758/s13414-018-1609-5

Palmisano, S., Gillam, B., Govan, D. G., Allison, R. S., and Harris, J. M. (2010). Stereoscopic perception of real depths at large distances. J. Vis. 10, 19-19. doi: 10.1167/10.6.19

Pelah, A., Barbur, J., Thurrell, A., and Hock, H. S. (2015). The coupling of vision with locomotion in cortical blindness. Vis. Res. 110(Pt B), 286-294. doi: 10. 1016/j.visres.2014.04.015

Pelah, A., and Barlow, H. B. (1996). Visual illusion from running. Nature 381:283. doi: $10.1038 / 381283 \mathrm{a} 0$

Powell, W., Stevens, B., Hand, S., and Simmonds, M. (2011). "Blurring the boundaries: The perception of visual gain in treadmill-mediated virtual environments," in Proceedings of the 3rd IEEE VR 2011 Workshop on Perceptual Illusions in Virtual Environments, Singapore.

Pretto, P., Bresciani, J.-P., Rainer, G., and Bülthoff, H. H. (2012). Foggy perception slows us down. eLife 1:e00031. doi: 10.7554/eLife.00031

Pretto, P., Ogier, M., Bülthoff, H. H., and Bresciani, J.-P. (2009). Influence of the size of the field of view on motion perception. Comput. Graph. 33, 139-146. doi: 10.1016/j.cag.2009.01.003

Riley, P. O., Dicharry, J., Franz, J., Della Croce, U., Wilder, R. P., and Kerrigan, D. C. (2008). A kinematics and kinetic comparison of overground and treadmill running. Med. Sci. Sports Exerc. 40, 1093-1100. doi: 10.1249/MSS. 0b013e3181677530

Souman, J. L., Frissen, I., Sreenivasa, M. N., and Ernst, M. O. (2009). Walking straight into circles. Curr. Biol. 19, 1538-1542. doi: 10.1016/j.cub.2009. 07.053

Sun, H.-J., Campos, J. L., and Chan, G. S. W. (2004a). Multisensory integration in the estimation of relative path length. Exp. Brain Res. 154, 246-254. doi: 10.1007/s00221-003-1652-9

Sun, H.-J., Campos, J. L., Young, M., Chan, G. S. W., and Ellard, C. G. (2004b). The contributions of static visual cues, nonvisual cues, and optic flow in distance estimation. Perception 33, 49-66. doi: 10.1068/p5145 
Thurrell, A., and Pelah, A. (2005). "Matching visual and nonvisual signals: evidence for a mechanism to discount optic flow during locomotion," in Proceedings of the Human Vision and Electronic Imaging X, Cambridge.

Thurrell, A. E. I., and Pelah, A. (2002). Reduction of perceived visual speed during walking: effect dependent upon stimulus similarity to the visual consequences of locomotion. J. Vis. 2, 628-628. doi: 10.1167/2.7.628

Thurrell, A. E. I., Pelah, A., and Distler, H. K. (1998). The influence of nonvisual signals of walking on the perceived speed of optic flow. Perception 27, $147-148$.

Turano, K. A., Yu, D., Hao, L., and Hicks, J. C. (2005). Optic-flow and egocentricdirection strategies in walking: central vs peripheral visual field. Vis. Res. 45, 3117-3132. doi: 10.1016/j.visres.2005.06.017

Van Veen, H. A. H. C., Distler, H. K., Braun, S. J., and Bülthoff, H. H. (1998). Navigating through a virtual city: using virtual reality technology to study human action and perception. Future Gener. Comput. Syst. 14, 231-242. doi: 10.1016/s0167-739x(98)00027-2

Wank, V., Frick, U., and Schmidtbleicher, D. (1998). Kinematics and electromyography of lower limb muscles in overground and treadmill running. Int. J. Sports Med. 19, 455-461. doi: 10.1055/s-2007-971944

Warren, W. H., and Hannon, D. J. (1988). Direction of self-motion is perceived from optical flow. Nature 336, 162-163. doi: 10.1038/336162a0
White, S. C., Yack, H. J., Tucker, C. A., and Lin, H.-Y. (1998). Comparison of vertical ground reaction forces during overground and treadmill walking. Med. Sci. Sports Exerc. 30, 1537-1542. doi: 10.1097/00005768-19981000000011

Yardley, L., and Higgins, M. (1998). Spatial updating during rotation: the role of vestibular information and mental activity. J. Vestib. Res. 8, 435-442.

Conflict of Interest: PP was employed by Virtual Vehicle Research Center, Graz, Austria.

The remaining authors declare that the research was conducted in the absence of any commercial or financial relationships that could be construed as a potential conflict of interest.

Copyright (c) 2019 Caramenti, Pretto, Lafortuna, Bresciani and Dubois. This is an open-access article distributed under the terms of the Creative Commons Attribution License (CC BY). The use, distribution or reproduction in other forums is permitted, provided the original author(s) and the copyright owner(s) are credited and that the original publication in this journal is cited, in accordance with accepted academic practice. No use, distribution or reproduction is permitted which does not comply with these terms. 